\title{
On the Geometry of Point-Plane Constraints on Rigid-Body Displacements
}

\author{
J.M. Selig
}

Received: date / Accepted: date

\begin{abstract}
In this paper the rigid-body displacements that transform a point in such a way that it remains on a particular plane are studied. These sets of rigid displacements are referred to as point-plane constraints and are given by the intersection of the Study quadric of all rigid displacements with another quadric in 7-dimensional projective space. The set of all possible point-plane constraints comprise a Segre variety. Two different classes of problems are investigated. First instantaneous kinematics, for a given rigid motion there are points in space which, at some instant, have no torsion or have no curvature to some order. The dimension and degrees of these varieties are found by very simple computations. The corresponding problems for point-sphere constraints are also found. The second class of problems concern the intersections of several given constraints. Again the characteristics of these varieties for different numbers of constraints are found using very simple techniques.
\end{abstract}

Keywords Rigid-body displacements · constraints · instantaneous kinematics · homology

PACS $70 \mathrm{~B} 10 \cdot 53 \mathrm{~A} 17 \cdot 14 \mathrm{~N} 25$

\section{Introduction}

This work is concerned with constraints on the displacements of a rigid body. In particular with constraints that require a point in the body to remain in a fixed plane. However, constraints where a point is required to remain on a fixed sphere will be briefly considered too. These will be called point-plane or point-sphere constraints respectively. Wampler [10] argued that the point-plane constraints arise in many area of kinematics and robotics. As examples he cites the kinematics of certain linkages, problems in metrology concerned with locating the position and orientation of a rigid body and also problems in robot vision where feature points are restricted to certain lines.

To these problems another is contributed here: instantaneous kinematics. The previous problems all reduce to finding the intersection of several given point-plane constraints. That

J.M. Selig

London South Bank University, London, U.K.

Tel.: +(0)20-7815-7461

E-mail: seligjm@1sbu.ac.uk 
is the rigid-body displacements which satisfy all the constraints. For instantaneous kinematics the problem is, in a sense, dual to this problem. Here the motion is given and the points which remain on some plane or sphere are sought. These problems can be answered by studying the spaces of all possible point-plane or point-sphere constraints.

Most of the examples considered in this work are not new and in some cases date back to the late 19th century. However, the methods used in this work are novel, at least in kinematics and robotics. The techniques used come from algebraic geometry, in particular homology is used as a generalisation of degree and Bézout's theorem to varieties such as quadrics and products of projective spaces. An excellent introduction to these methods is [3]. In other work, for example see [5], much more advanced methods from algebraic geometry are used. The idea here is to keep the use of advanced techniques to a bare minimum. This is partly to show how far we can get with ideas that are only a little more advanced than those commonly used. The main motivation though, is to show that these methods are simple to use and provide useful new information about important problems in robotics and mechanisms theory.

In a previous work [8], a particular Clifford algebra was used to compare point-plane, point-sphere and some other geometric constraints. The present work concentrates on the geometry of the constraints so dual quaternions are used here to reprove that point-plane and point-sphere constraints are represented by the intersection of the Study quadric with another quadric hypersurface in $\mathbb{P}^{7}$. The following section gives a brief introduction to the Study quadric and dual quaternions.

\section{Dual Quaternions and the Study quadric}

A dual quaternion is an element of the algebra $\mathbb{H} \otimes \mathbb{D}$, the tensor product of the quaternions with the ring of dual numbers. A typical dual quaternion has the form, $a+\varepsilon c$ where $a$ and $c$ are quaternions and $\varepsilon$ is the dual unit which commutes with scalars and quaternions and squares to zero, $\varepsilon^{2}=0$. The quaternions have the form,

$$
a=a_{0}+a_{1} i+a_{2} j+a_{3} k
$$

where $i, j$ and $k$ are quaternion generators which satisfy $i^{2}=j^{2}=k^{2}=-1, i j=k, j k=i$, $k i=j$ and so on.

It is well known that rotations can be represented by unit quaternions. A unit quaternion $r=a_{0}+a_{1} i+a_{2} j+a_{3} k$, satisfies the equation, $r r^{-}=1$ that is $a_{0}^{2}+a_{1}^{2}+a_{2}^{2}+a_{3}^{2}=1$, since the quaternion conjugate is given by $r^{-}=a_{0}-a_{1} i-a_{2} j-a_{3} k$. If $p=x i+y j+z k$ is a pure quaternion representing a point in space, then a rotation is given by a product of the form,

$$
p^{\prime}=r p r^{-} .
$$

Both $r$ and $-r$ give the same rotation so the unit quaternions represent a double cover of the group of rotations about the origin.

To represent rigid displacements the dual numbers are introduced. Now a point in space is represented by a dual quaternion of the form $1+\varepsilon p$, with $p$ as above. Rotations are represented by unit quaternions again but translations are represented by dual quaternions of the form $1+(1 / 2) \varepsilon\left(t_{x} i+t_{y} j+t_{z} k\right)$. In this representation $t_{x}, t_{y}$ and $t_{z}$ are the components of the translation vector. A general dual quaternion acts on points in space according to the relation,

$$
1+\varepsilon p^{\prime}=(a+\varepsilon c)(1+\varepsilon p)\left(a^{-}-\varepsilon c^{-}\right) .
$$


Notice, that both $(a+\varepsilon c)$ and $-(a+\varepsilon c)$ give the same rigid displacement. Not every dual quaternion represents a rigid displacement, first it must satisfy $a a^{-}=1$ that is,

$$
a_{0}^{2}+a_{1}^{2}+a_{2}^{2}+a_{3}^{2}=1 .
$$

Second it must satisfy $a c^{-}+c a^{-}=0$, to see this observe that $t=(1 / 2) c a^{-}$and since $t$ has no scalar part $t+t^{-}=0$. In coordinates this equation becomes,

$$
a_{0} c_{0}+a_{1} c_{1}+a_{2} c_{2}+a_{3} c_{3}=0 .
$$

To get rid of the double valued nature of this representation $\left(a_{0}: a_{1}: a_{2}: a_{3}: c_{0}: c_{1}: c_{2}: c_{3}\right)$ are taken as homogeneous coordinates in $\mathbb{P}^{7}$. Points in $\mathbb{P}^{7}$ are rays through the origin in $\mathbb{R}^{8}$ (in the following the scalars will often implicitly be assumed to be complex rather than real to make degree computations simpler). Almost all of these rays will meet the affine quadric given by (1) in the two points representing the same rotation. So only the second equation (2) is relevant. This is a homogeneous quadratic equation, the solution to this equation form a six-dimensional quadric in $\mathbb{P}^{7}$ known as the Study quadric. Almost all points of this quadric are in one-to-one correspondence with the group of proper rigid-body displacements, that is the Lie group $S E(3)$. The exceptions to this correspondence consist of a single 3-plane of elements satisfying $a_{0}=a_{1}=a_{2}=a_{3}=0$. This 3-plane of 'ideal element' or 'elements at infinity' will be referred to as $A_{\infty}$ in the following.

More details on these ideas can be found in [7].

\section{Quadratic Constraints}

\subsection{A Point on a Plane}

The standard vector equation for a point $\mathbf{p}$ to lie on a plane with normal vector $\mathbf{n}$ is,

$$
\mathbf{n} \cdot \mathbf{p}=\delta
$$

where $\delta$ is a real constant. If the point lies on the plane initially then it remains on the plane after a rigid displacement if,

$$
\mathbf{n} \cdot(R \mathbf{p}+\mathbf{t}-\mathbf{p})=0,
$$

where as usual, $R$ is a $3 \times 3$ rotation matrix and $\mathbf{t}$ a translation vector.

Proposition 1 The rigid body displacements which preserve the incidence of a point with a plane comprise an open set of a quadric hypersurface in $\mathbb{P}^{7}$.

Proof In the quaternion representation introduced above, the effect of a rotation can be written $a p a^{-}$and the pure quaternion representing a translation is $2 c a^{-}$or $-2 a c^{-}$. Now using the fact that $a a^{-}=1$, the equation for the plane can be written,

$$
n\left(a p a^{-}-p a a^{-}+2 c a^{-}\right)^{-}+\left(a p a^{-}-p a a^{-}+2 c a^{-}\right) n^{-}=0
$$

or more compactly as,

$$
\operatorname{Re}\left(\left(a p a^{-}-p a a^{-}+2 c a^{-}\right) n^{-}\right)=0
$$

where $\operatorname{Re}()$ denotes the real (scalar) part of the quaternion. Notice that given a point $p$ and a normal $n$ this equation is quadratic in the components of the dual quaternion $a+\varepsilon c$. 
Remark 1 This equation can be put in the matrix form,

$$
\mathbf{g}^{T} \mathrm{Q}_{\pi}(p, n) \mathbf{g}=0,
$$

where the possible group elements are given by $\mathbf{g}^{T}=\left(a_{0}, a_{1}, a_{2}, a_{3}, c_{0}, c_{1}, c_{2}, c_{3}\right)$ the components of the dual quaternion, and the elements of the symmetric $8 \times 8$ matrix $\mathrm{Q}_{\pi}(p, n)$ can be found by direct computation. In fact it makes no difference if we use the negative of the matrix computed since the equation is homogeneous, however the form given below will make things a little simpler latter.

The symmetric matrix is most conveniently expressed in a partitioned form,

$$
\mathrm{Q}_{\pi}(p, n)=\left(\begin{array}{cc}
\mathscr{W} & \mathscr{N} \\
\mathscr{N}^{T} & 0
\end{array}\right)
$$

where

$$
\mathscr{W}=\left(\begin{array}{cccc}
0 & n_{2} p_{3}-n_{3} p_{2} & n_{3} p_{1}-n_{1} p_{3} & n_{1} p_{2}-n_{2} p_{1} \\
n_{2} p_{3}-n_{3} p_{2} & 2\left(n_{2} p_{2}+n_{3} p_{3}\right) & -\left(n_{2} p_{1}+n_{1} p_{2}\right) & -\left(n_{3} p_{1}+n_{1} p_{3}\right) \\
n_{3} p_{1}-n_{1} p_{3} & -\left(n_{2} p_{1}+n_{1} p_{2}\right) & 2\left(n_{1} p_{1}+n_{3} p_{3}\right) & -\left(n_{3} p_{2}+n_{2} p_{3}\right) \\
n_{1} p_{2}-n_{2} p_{1} & -\left(n_{3} p_{1}+n_{1} p_{3}\right) & -\left(n_{3} p_{2}+n_{2} p_{3}\right) & 2\left(n_{1} p_{1}+n_{2} p_{2}\right)
\end{array}\right)
$$

and

$$
\mathscr{N}=\left(\begin{array}{cccc}
0 & -n_{1} & -n_{2} & -n_{3} \\
n_{1} & 0 & -n_{3} & n_{2} \\
n_{2} & n_{3} & 0 & -n_{1} \\
n_{3} & -n_{2} & n_{1} & 0
\end{array}\right)
$$

These $4 \times 4$ matrices can in turn be partitioned as follows,

$$
\mathscr{W}=\left(\begin{array}{cc}
0 & (\mathbf{n} \times \mathbf{p})^{T} \\
(\mathbf{n} \times \mathbf{p}) & P N^{T}+N P^{T}
\end{array}\right),
$$

and

$$
\mathscr{N}=\left(\begin{array}{cc}
0 & -\mathbf{n}^{T} \\
\mathbf{n} & N
\end{array}\right) .
$$

where, $P$ is the $3 \times 3$ anti-symmetric matrix corresponding to the vector $\mathbf{p}$ and $N$ the antisymmetric matrix for $\mathbf{n}$.

\subsection{The Space of all Pointed Planes}

In the previous section a general result was derived for the quadric in $\mathbb{P}^{7}$ satisfied by all rigid displacements which preserve the incidence of an arbitrary point $\mathbf{p}$ with a plane with normal vector $\mathbf{n}$. In this section the space of all such quadrics will be investigated. Since each of these quadrics corresponds to a unique plane containing a particular point, the set of all such quadrics corresponds to the space of all pointed planes in space.

Proposition 2 The space of all pointed planes is isomorphic to an open set in the Segre variety $\mathbb{P}^{2} \times \mathbb{P}^{3}$. 
Proof Suppose, $\mathbf{n}=\left(n_{1}, n_{2}, n_{3}\right)^{T}$ is the normal to the plane, clearly multiplying this vector by a non-zero scalar will not affect the solutions of the quadratic equation determined by a quadric. Also the components of $\mathbf{n}$ cannot all be zero, so we can assume that the components $\left(n_{1}: n_{2}: n_{3}\right)$ are homogeneous coordinates for a 2-dimensional projective space $\mathbb{P}^{2}$. Next we can assume that the components of the point are given by $\mathbf{p}=\left(p_{1}, p_{2}, p_{3}\right)^{T}$. Let us introduce another quantity $p_{0}$ and use this to multiply the top right and bottom left blocks of $\mathrm{Q}_{\pi}(p, n)$, see (3). Now the components $\left(p_{0}: p_{1}: p_{2}: p_{3}\right)$ can be taken as homogeneous coordinates in a $\mathbb{P}^{3}$. This does however introduce a set of ideal pointed planes, the set of quadrics with $p_{0}=0$. The elements of the matrix $\mathrm{Q}_{\pi}(p, n)$ are now linear combinations of elements of the form $p_{i} n_{j}$ with $0 \leq i \leq 3$ and $1 \leq j \leq 3$. Clearly, there are 12 different such elements and among the components of $\mathrm{Q}_{\pi}(p, n)$ can be found 12 linearly independent combinations. The twelve elements $p_{i} n_{j}$ can be thought of as homogeneous coordinates in a $\mathbb{P}^{11}$ but not all points in this projective space correspond to pointed planes, only those with coordinates which can be decomposed into products of $p_{i}$ s with $n_{j} \mathrm{~s}$. This is a well known construction in algebraic geometry known as a Segre variety. It is the image of $\mathbb{P}^{2} \times \mathbb{P}^{3}$ in $\mathbb{P}^{11}$.

This Segre variety is five dimensional and has degree $\left(\begin{array}{c}2+3 \\ 2\end{array}\right)=10$, see [3, $\left.\S 18.15\right]$. Also according to Harris $[3, \S 9.21]$, the lines in this Segre variety form two families, one family are the images of lines in $\mathbb{P}^{2}$ and the other family come from lines in $\mathbb{P}^{3}$.

Corollary 1 Lines in the Segre variety $\mathbb{P}^{2} \times \mathbb{P}^{3}$ correspond to pencils of quadrics which either represent pointed lines or points on parallel planes.

Proof Consider the intersection of two quadrics $Q_{\pi}\left(\mathbf{p}, \mathbf{n}_{1}\right)$ and $Q_{\pi}\left(\mathbf{p}, \mathbf{n}_{2}\right)$. The intersection will consist of displacements which preserve the incidence of the point $\mathbf{p}$ with two different planes with normals $\mathbf{n}_{1}$ and $\mathbf{n}_{2}$, in other words the set of displacement which leave the point on a line. The line being the intersection of the planes. From the structure of the matrices given in (3) above, it is clear that,

$$
\lambda \mathrm{Q}_{\pi}\left(\mathbf{p}, \mathbf{n}_{1}\right)+\mu \mathrm{Q}_{\pi}\left(\mathbf{p}, \mathbf{n}_{2}\right)=\mathrm{Q}_{\pi}\left(\mathbf{p}, \lambda \mathbf{n}_{1}+\mu \mathbf{n}_{2}\right),
$$

for arbitrary scalars $\lambda, \mu$. That is the linear system of quadrics determined by the two original quadric lies entirely in the Segre variety. Another way of putting this is that the pencil of quadrics determined by a point on a line in space is given by a line in the Segre variety. This is not the case for two general quadrics $Q_{\pi}\left(\mathbf{p}_{1}, \mathbf{n}_{1}\right)$ and $Q_{\pi}\left(\mathbf{p}_{2}, \mathbf{n}_{2}\right)$, the pencil determined by these quadrics will be a line in $\mathbb{P}^{11}$. However, it is also easy to see that for a pair of points restricted to parallel planes the line again lies in the Segre variety.

Remark 2 Finally here it is not hard to see that the set of displacements which lie in the intersection of three quadrics determined by three non-collinear points restricted to parallel planes actually form a subgroup of the group of rigid-body displacements. This subgroup is isomorphic to the group of proper planar rigid displacements: $S E(2)$ if two or all three planes are different. If all three parallel planes coincide then reflections in lines lying in the plane are also allowed, so the relevant subgroup is $E(2)$ in these cases.

\subsection{A Point on a Sphere}

For a point $\mathbf{p}$ to lie on a sphere with centre $\mathbf{q}$ and radius $r$ the equations is,

$$
(\mathbf{p}-\mathbf{q}) \cdot(\mathbf{p}-\mathbf{q})=r^{2}
$$


If the point already lies on the sphere the rigid displacements that keep it on the sphere must satisfy,

$$
(R \mathbf{p}+\mathbf{t}-\mathbf{q}) \cdot(R \mathbf{p}+\mathbf{t}-\mathbf{q})=(\mathbf{p}-\mathbf{q}) \cdot(\mathbf{p}-\mathbf{q})
$$

Proposition 3 The rigid body displacements which preserve the incidence of a point with a sphere comprise an open set of a quadric hypersurface in $\mathbb{P}^{7}$.

Proof The equation above can be written using quaternions as;

$$
\left(a p a^{-}+2 c a^{-}-q\right)\left(a p a^{-}+2 c a^{-}-q\right)^{-}=(p-q)(p-q)^{-}=p p^{-}-p q^{-}-q p^{-}+q q^{-} .
$$

Expanding and making the equation homogeneous using $a a^{-}=1$ leads to,

$a a^{-}\left(p q^{-}+q p^{-}\right)-a p a^{-} q^{-}-q a p^{-} a^{-}+2\left(c p^{-} a^{-}+a p c^{-}\right)-2\left(q a c^{-}+c a^{-} q^{-}\right)+4 c c^{-}=0$.

Again this can be written as a quadratic in the coordinates of $\mathbb{P}^{7}$.

Remark 3 In matrix form this is,

$$
\mathbf{g}^{T} \mathrm{Q}_{S}(p, q) \mathbf{g}=0
$$

The $8 \times 8$ symmetric matrix $\mathrm{Q}_{S}(p, q)$ can be partitioned as,

$$
\mathrm{Q}_{S}(p, q)=\left(\begin{array}{cc}
\mathscr{U} & \mathscr{P}+\mathscr{Q} \\
\mathscr{P}^{T}+\mathscr{Q}^{T} & 2 I
\end{array}\right)
$$

where,

$$
\mathscr{U}=\left(\begin{array}{cc}
0 & (\mathbf{q} \times \mathbf{p})^{T} \\
(\mathbf{q} \times \mathbf{p}) & P Q^{T}+Q P^{T}
\end{array}\right),
$$

and $\mathscr{Q}$ is similar to $\mathscr{N}$ above,

$$
\mathscr{Q}=\left(\begin{array}{cc}
0 & -\mathbf{q}^{T} \\
\mathbf{q} & Q
\end{array}\right)
$$

but $\mathscr{P}$ is slightly different,

$$
\mathscr{P}=\left(\begin{array}{cc}
0 & \mathbf{p}^{T} \\
-\mathbf{p} & P
\end{array}\right),
$$

the difference being a change of sign in the first row and first column. Note that these results agree with those given by Wampler [9] and Husty [4].

Proposition 4 The space of pointed spheres is an open set in the Segre variety $\mathbb{P}^{3} \times \mathbb{P}^{3}$

Proof We can introduce homogonising variables $p_{0}$ and $q_{0}$ and then write the matrix of the quadric as,

$$
\mathrm{Q}_{S}(p, q)=\left(\begin{array}{cc}
\mathscr{U} & \mathscr{P} q_{0}+p_{0} \mathscr{Q} \\
\mathscr{P}^{T} q_{0}+p_{0} \mathscr{Q}^{T} & 2 p_{0} q_{0} I
\end{array}\right)
$$

As usual this introduces ideal points, but here it can be seen that if $q_{0}=0$ the quadric is the constraint quadric for a pointed plane with normal q. Arguing in an analogous fashion to the proof of proposition 2 we have the result. 


\section{Infinitesimal Kinematics}

The results of this section are classical, many of them seem to be due to Schönflies [6]. The novelty here is the simplicity of the proofs which depend on propositions 2 and 4 above.

Corollary 2 There are, in general, 10 points which in 6 positions lie in a plane.

Proof The six positions refer to positions of a rigid body. These can be thought of as a home position and five rigid displacements $g_{1}, g_{2}, g_{3}, g_{4}, g_{5}$. These displacements will move the home position to one of the five other positions. On the other hand we might consider successive displacements from one position to the next; such displacements would be given by $g_{1}, g_{2} g_{1}^{-1}, g_{3} g_{2}^{-1}, \ldots, g_{5} g_{4}^{-1}$. In either case, we may consider the points which remain on a plane after a particular displacement. These points and planes are determined by the quadrics. If,

$$
\mathbf{g}_{i}^{T} \mathrm{Q}_{\pi}(p, n) \mathbf{g}_{i}=0
$$

then the quadric $\mathrm{Q}_{\pi}(p, n)$ represents a point on a plane which remains on the plane after the displacement $g_{i}$. Notice that this equation is linear in the elements of the symmetric matrix $\mathrm{Q}_{\pi}(p, n)$, hence such an equation determines a hyperplane in the $\mathbb{P}^{11}$ of homogeneous coordinates $p_{i} n_{j}$. The intersection of five such hyperplanes will, in general be a sixdimensional plane in $\mathbb{P}^{11}$. Since the Segre variety is 5 dimensional, the intersection with a six-dimensional plane will give a finite number of points. Ten intersections in general, since the degree of the Segre variety is 10 .

Results on finite numbers of positions were originally intended to produce results about infinitesimal motion after a suitable limiting process. These problems are considered next.

\subsection{Contact with Planes}

Let $g(t)$ be an analytic rigid-body motion. The Taylor expansion of the motion about the point $g(0)=g_{0}$ is given by,

$$
g(t)=g_{0}+\frac{d g_{0}}{d t} t+\frac{d^{2} g_{0}}{d t^{2}} \frac{t^{2}}{2}+\frac{d^{3} g_{0}}{d t^{3}} \frac{t^{3}}{3 !}+\cdots .
$$

The corresponding Lie algebra element $s$ is given by $s=(d g / d t) g^{-1}$ and so the first few derivatives can be written,

$$
\frac{d g}{d t}=s g, \quad \frac{d^{2} g}{d t^{2}}=\left(\dot{s}+s^{2}\right) g, \quad \frac{d^{3} g}{d t^{3}}=\left(\ddot{s}+2 \dot{s} s+s \dot{s}+s^{3}\right) g, \quad \ldots
$$

The Lie algebra element $s$ and its derivatives are to be treated as dual quaternions in the above. We can choose the coordinate frame so that $g(0)=1$, the identity in the group, this will not affect the results here. Expressions will be simplified if we set,

$$
z_{0}=1, \quad z_{1}=s, \quad z_{2}=\left(\dot{s}+s^{2}\right), \quad z_{3}=\left(\ddot{s}+2 \dot{s} s+s \dot{s}+s^{3}\right), \quad \ldots
$$

The Taylor expansion can then be written as,

$$
g(t)=z_{0}+z_{1} t+z_{2} \frac{t^{2}}{2}+z_{3} \frac{t^{3}}{3 !}+\cdots
$$


Substituting this into the quadric equation and treating the dual quaternions as vectors now gives,

$$
\mathbf{g}^{T}(t) \mathrm{Q}_{\pi}(p, n) \mathbf{g}(t)=\sum_{i=0}^{\infty} t^{i}\left(\sum_{j=0}^{i} \frac{1}{j !(i-j) !} \mathbf{z}_{i}^{T} \mathrm{Q}_{\pi}(p, n) \mathbf{z}_{i-j}\right) .
$$

For the motion to move a point entirely within a plane the curve $\mathbf{g}(t)$ must lie in the quadric for all $t$. However, if we are only interested in the infinitesimal properties of the motion at $t=0$ we can ask if a point remains on a plane up to some particular order. This would require that the coefficients of $t^{i}$ in the expression above, all vanish up to some value of $i$. For example the points which lie on a plane up to order 3 are determined by the 3 equations,

$$
\begin{aligned}
& 0=\mathbf{z}_{0}^{T} \mathrm{Q}_{\pi}(p, n) \mathbf{z}_{1}+\mathbf{z}_{1}^{T} \mathrm{Q}_{\pi}(p, n) \mathbf{z}_{0}, \\
& 0=\mathbf{z}_{0}^{T} \mathrm{Q}_{\pi}(p, n) \mathbf{z}_{2}+2 \mathbf{z}_{1}^{T} \mathrm{Q}_{\pi}(p, n) \mathbf{z}_{1}+\mathbf{z}_{2}^{T} \mathrm{Q}_{\pi}(p, n) \mathbf{z}_{0}, \\
& 0=\mathbf{z}_{0}^{T} \mathrm{Q}_{\pi}(p, n) \mathbf{z}_{3}+3 \mathbf{z}_{1}^{T} \mathrm{Q}_{\pi}(p, n) \mathbf{z}_{2}+3 \mathbf{z}_{2}^{T} \mathrm{Q}_{\pi}(p, n) \mathbf{z}_{1}+\mathbf{z}_{3}^{T} \mathrm{Q}_{\pi}(p, n) \mathbf{z}_{0} .
\end{aligned}
$$

The first possible equation $0=\mathbf{z}_{0}^{T} \mathrm{Q}_{\pi}(p, n) \mathbf{z}_{0}$, is automatically satisfied since $z_{0}=1$ and the identity lies on every quadric. Notice that the three equations given above are all linear in the elements $p_{i} n_{j}$ of the quadrics, in fact they are linear in both the $p_{i}$ variables and the $n_{j}$ variable. That is, the equation are multi-homogeneous with bi-degree $(1,1)$. Geometrically this can be thought of as pulling the equations back to $\mathbb{P}^{2} \times \mathbb{P}^{3}$ using the Segre map.

Intersections in $\mathbb{P}^{2} \times \mathbb{P}^{3}$ can be studied using homology, see $[7, \S 11.5]$ for example ${ }^{1}$. The homology of $\mathbb{P}^{2} \times \mathbb{P}^{3}$ is generated by elements $\alpha_{i} \beta_{j}$, where $0 \leq i \leq 2$ and $0 \leq j \leq 3$. Here $\alpha_{i}$ is the class of an $i$-plane in $\mathbb{P}^{2}$ and $\beta_{j}$ the class of a $j$-plane in $\mathbb{P}^{3}$. The intersection of these classes is given by $\left(\alpha_{i} \beta_{j}\right) \cap\left(\alpha_{k} \beta_{l}\right)=\alpha_{i+k-2} \beta_{j+l-3}$ when $0 \leq i+k-2 \leq 2$ and $0 \leq j+l-3 \leq 3$ and zero otherwise.

Corollary 3 The points in space which, at some instant, have no torsion lie on a cubic surface.

Proof If a point has no torsion instantaneously then its velocity, acceleration and jerk must be coplanar at the instant in question. This means that the point must have contact with a plane up to third order. This gives three linear conditions on the quadrics or three $(1,1)$ polynomial in the Segre variety.

The homology class of a $(1,1)$-polynomial is $\alpha_{1} \beta_{3}+\alpha_{2} \beta_{2}$ and the intersection of three such polynomials gives a variety of class,

$$
\left(\alpha_{1} \beta_{3}+\alpha_{2} \beta_{2}\right)^{3}=\left(\alpha_{0} \beta_{3}+2 \alpha_{1} \beta_{2}+\alpha_{2} \beta_{1}\right) \cap\left(\alpha_{1} \beta_{3}+\alpha_{2} \beta_{2}\right)=3 \alpha_{0} \beta_{2}+3 \alpha_{1} \beta_{1}+\alpha_{2} \beta_{0} .
$$

Projecting to the $\mathbb{P}^{3}$ component gives a variety with homology class $3 \beta_{2}$, representing a cubic surface.

Corollary 4 The points in space which, at some instant, have fourth order contact with a plane lie on a curve of degree six.

Proof If we looking at points which lie on a plane up to order 4 then we must intersect 4 $(1,1)$-polynomials,

$$
\left(\alpha_{1} \beta_{3}+\alpha_{2} \beta_{2}\right)^{4}=6 \alpha_{0} \beta_{1}+4 \alpha_{1} \beta_{0} .
$$

Projecting to $\mathbb{P}^{3}$ gives $6 \beta_{1}$, the homology class of a degree 6 curve.

\footnotetext{
1 Notice that the '2-homogeneous Bezout numbers' used by Wampler [9] are essentially cohomology classes.
} 
Corollary 5 The are generally 10 points in space which, at some instant, have fifth order contact with a plane.

Proof This is essentially the first result in this section,

$$
\left(\alpha_{1} \beta_{3}+\alpha_{2} \beta_{2}\right)^{5}=10 \alpha_{0} \beta_{0} .
$$

\subsection{Contact with Spheres}

The arguments of the previous section can be repeated with points on spheres rather than planes. The space of all pointed spheres is an open set in $\mathbb{P}^{3} \times \mathbb{P}^{3}$. The homology of $\mathbb{P}^{3} \times \mathbb{P}^{3}$ is generated by elements $\alpha_{i} \beta_{j}$, where $0 \leq i \leq 3$ and $0 \leq j \leq 3$. Here $\alpha_{i}$ is the class of an $i$-plane in $\mathbb{P}^{3}$ and $\beta_{j}$ the class of a $j$-plane in the other $\mathbb{P}^{3}$. The intersection of these classes is given by $\left(\alpha_{i} \beta_{j}\right) \cap\left(\alpha_{k} \beta_{l}\right)=\alpha_{i+k-3} \beta_{j+l-3}$ when $0 \leq i+k-3 \leq 3$ and $0 \leq j+l-3 \leq 3$ and zero otherwise. The homology class of a $(1,1)$-polynomial is $\alpha_{2} \beta_{3}+\alpha_{3} \beta_{2}$.

Corollary 6 The points in space which, at some instant, have fourth order contact with a sphere lie on a quartic surface. The centres of the corresponding spheres also lie on a quartic surface.

Proof The intersection of four $(1,1)$-polynomials gives a variety of class,

$$
\left(\alpha_{2} \beta_{3}+\alpha_{3} \beta_{2}\right)^{4}=4 \alpha_{0} \beta_{2}+6 \alpha_{1} \beta_{1}+4 \alpha_{2} \beta_{0} .
$$

Projecting to the first $\mathbb{P}^{3}$ component gives a variety with homology class $4 \beta_{2}$, representing a quartic surface. The points on this surface are points where the first four derivatives of position instantaneously agree with a motion on a sphere. That is, points which have fourth order contact with a sphere. On the other hand if we project to the other $\mathbb{P}^{3}$ factor we get $4 \alpha_{2}$, also corresponding to a quartic surface. This time the surface consists of the centres of the spheres which contact the trajectories of point to forth order.

Corollary 7 The points in space which, at some instant, have fifth order contact with a sphere lie on a curve of degree 10, as do the centres of the spheres.

Proof Looking at fifth order contact we see that,

$$
\left(\alpha_{2} \beta_{3}+\alpha_{3} \beta_{2}\right)^{5}=10 \alpha_{0} \beta_{1}+10 \alpha_{1} \beta_{0} .
$$

Showing that the points which instantaneously have order 5 contact with spheres lie on a curve of degree 10 , as do the centres of the spheres.

Corollary 8 The are generally 20 points in space which, at some instant, have sixth order contact with a sphere.

Proof Now,

$$
\left(\alpha_{2} \beta_{3}+\alpha_{3} \beta_{2}\right)^{6}=20 \alpha_{0} \beta_{0},
$$

showing that in general there are 20 points with order 6 contact with a sphere. 


\section{Intersection of Point-Line Constraints}

In this section the problem of finding the possible motions of a rigid-body with one or more points constrained to lie on given lines is addressed. Clearly the constraints for a single point to lie on a line can be expressed as a pair of point-plane constraints where the same point is constrained to a pair of planes intersecting along the given line. So it might be thought that this problem is simply a special case of the examples considered below. However, as will be shown, this is not exactly what happens.

\subsection{A Point Constrained by a Line}

Rather than looking at the constraint varieties that such a subspace must lie on, let us think about how this subspace could be parameterised.

Proposition 5 The group elements which preserve the incidence of a point with a line form $a \mathbb{P}^{1} \times \mathbb{P}^{3}$ Segre variety in the Study quadric.

Proof Consider the example here of a point at the origin constrained to remain on the $x$ axis. Clearly any rotation about the origin will be such an element as will any translation along the line. Combining these gives a 4-dimensional set of group elements that can be parameterised as,

$$
g=\left(\lambda_{0}+\varepsilon \lambda_{1} i\right)\left(\mu_{0}+\mu_{1} i+\mu_{2} j+\mu_{3} k\right),
$$

where $\mu_{i}$ and $\lambda_{j}$ are arbitrary parameters. The parameters $\left(\lambda_{0}: \lambda_{1}\right)$ can be thought of as homogeneous coordinates for a $\mathbb{P}^{1}$ and $\left(\mu_{0}: \mu_{1}: \mu_{2}: \mu_{3}\right)$ for $\mathbb{P}^{3}$. The image in $\mathbb{P}^{7}$ is given in coordinates by $g=a+\varepsilon c$ with,

$$
\begin{aligned}
& a=\lambda_{0} \mu_{0}+\lambda_{0} \mu_{1} i+\lambda_{0} \mu_{2} j+\lambda_{0} \mu_{3} k, \\
& c=-\lambda_{1} \mu_{1}+\lambda_{1} \mu_{0} i-\lambda_{1} \mu_{3} j+\lambda_{1} \mu_{2} k .
\end{aligned}
$$

Finally, it is clear that any point on a line can be transformed to this configuration using a suitable conjugation in the group.

Remark 4 This Segre variety lies in several quadrics in $\mathbb{P}^{7}$, the equations of these quadrics can be found by asserting that the rank of the following matrix is 1 or less:

$$
\left(\begin{array}{cccc}
a_{0} & a_{1} & a_{2} & a_{3} \\
c_{1} & -c_{0} & c_{3} & -c_{2}
\end{array}\right)
$$

Ostensibly this will give 6 quadrics, however the variety will lie on any linear combination of these six. In particular, it is straightforward to check that the Study quadric and the quadrics $\mathrm{Q}_{\pi}(0, j)$ and $\mathrm{Q}_{\pi}(0, k)$, all lie in the the linear system formed by these quadrics.

Remark 5 According to Harris [3, p.233] the degree of such a Segre variety is $\left(\begin{array}{c}1+3 \\ 3\end{array}\right)=$ 4. This suggests that there is another component to the intersection of the 3 quadrics; $\mathrm{Q}_{\pi}(0, j), \mathrm{Q}_{\pi}(0, k)$ and the Study quadric. Consider the following parameterisation of a five dimensional variety,

$$
\begin{array}{ll}
a_{0}=\lambda_{0} \mu_{0}, & c_{0}=-\lambda_{1} \mu_{1}+\lambda_{2} \mu_{0}, \\
a_{1}=\lambda_{0} \mu_{1}, & c_{1}=\lambda_{1} \mu_{0}+\lambda_{2} \mu_{1}, \\
a_{2}=\lambda_{0} \mu_{2}, & c_{2}=-\lambda_{1} \mu_{3}+\lambda_{2} \mu_{2}, \\
a_{3}=\lambda_{0} \mu_{3}, & c_{3}=\lambda_{1} \mu_{2}+\lambda_{2} \mu_{3} .
\end{array}
$$




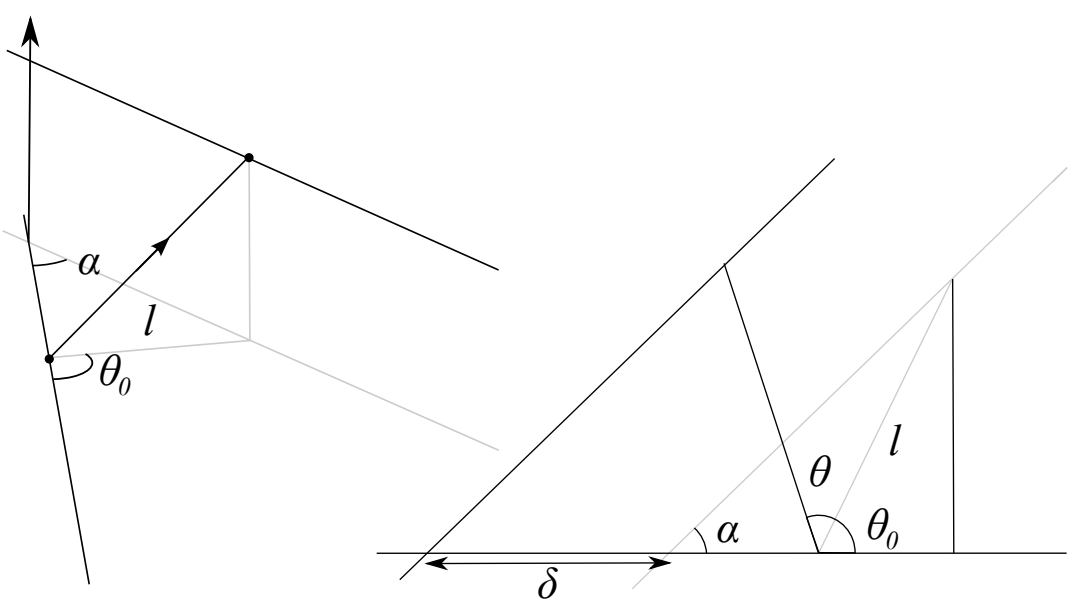

Fig. 1 Two points on two lines.

It is simple to check that this variety lies in both quadrics $\mathrm{Q}_{\pi}(0, j)$ and $\mathrm{Q}_{\pi}(0, k)$. However, substituting the parameterisation into the Study quadric gives,

$$
\lambda_{0} \lambda_{2}\left(\mu_{0}^{2}+\mu_{1}^{2}+\mu_{2}^{2}+\mu_{3}^{2}\right)
$$

Hence the intersection of all three quadrics consists of two 4-dimensional varieties. For the first $\lambda_{2}=0$ and the Segre variety discussed above is recovered. The second component arises when $\left(\mu_{0}^{2}+\mu_{1}^{2}+\mu_{2}^{2}+\mu_{3}^{2}\right)=0$. All solutions to this condition will be complex and are thus unphysical and can be safely ignored in the following. When $\lambda_{0}=0$ the result is the 3-plane $A_{\infty}$, which lies in the Segre variety.

\subsection{Two Point-Line Constraints}

The intersection of two Segre varieties as found in the previous section will give a 2dimensional variety representing the group elements which preserve the incidences of two points on a rigid body with a pair of lines. This variety can also be readily parametrised.

Proposition 6 The possible rigid-body displacements allowed by a body constrained in such a way that two of its points are restricted to move on a pair of lines, forms a 2 dimensional variety with degree 6.

Proof The possible displacements can be split into a rotation about the axis joining the two points followed by a displacement of the points along their lines. To understand the displacement of the points along the lines it is useful to project the lines into the plane perpendicular to the common normal to the lines, see figure 1 . This reduces the problem to a well known problem in planar kinematics. The motion of a rigid bar so that two of its point remain on a pair of given lines is know as Cardan motion, see [1, ch. 9 \$11]. This motion can be thought of as a rotation about the first point followed by a translation along the first line until the second point regains contact with its line. 
To summarise, any displacement which moves a pair of points in such a way that they remain on a pair of given lines can be written as the product of three dual quaternion factors. Using the coordinates given in figure 1 the factors can be written,

$$
g=\left(1+\varepsilon \frac{\delta}{2} i\right)(c+s k)\left(c^{\prime}+s^{\prime} \mathbf{p}_{12}\right)
$$

where $\mathbf{p}_{12}$ is the unit pure quaternion corresponding to the vector joining the two points, and $c^{\prime}=\cos (\phi / 2), s^{\prime}=\sin (\phi / 2)$ with $\phi$ the rotation angle about the line. The second factor gives a rotation about the vertical, the common perpendicular to the lines with $c=\cos (\theta / 2)$ and $s=\sin (\theta / 2)$. After a little trigonometry, the translation along the first axis is given by,

$$
\delta=2 l\left(\frac{\cos \left(\alpha-\theta_{0}\right)}{\sin \alpha}\right) s c+2 l\left(\frac{\sin \left(\alpha-\theta_{0}\right)}{\sin \alpha}\right) s^{2} .
$$

Now consider the 2 dimensional variety parameterised as above as a subvariety of the Segre variety formed by the first point moving on the first line. The bidegree of this subvariety can be found by projecting onto the two factors of the Segre variety. Projecting on to the $\mathbb{P}^{3}$ of rotations about the origin gives a two dimensional variety parameterised as $(c+s k)\left(c^{\prime}+s^{\prime} \mathbf{p}_{12}\right)$. This is again a product, this time $\mathbb{P}^{1} \times \mathbb{P}^{1}$; the standard quadric in $\mathbb{P}^{3}$. Projecting to the $\mathbb{P}^{1}$ of translations along the $x$-axis gives another degree 2 variety. Hence we can conclude that the homology of this subvariety is given by, $2 \alpha_{1} \beta_{1}+2 \alpha_{0} \beta_{2}$, where as usual the homology of $\mathbb{P}^{1} \times \mathbb{P}^{3}$ is generated by elements $\alpha_{i} \beta_{j}$, where $0 \leq i \leq 1$ and $0 \leq j \leq 3$.

As a subvariety of $\mathbb{P}^{7}$, the intersection of the two Segre varieties is a 2 -dimensional variety with degree,

$$
2\left(\begin{array}{l}
2 \\
0
\end{array}\right)+2\left(\begin{array}{l}
2 \\
1
\end{array}\right)=2 \times 1+2 \times 2=6
$$

see [3, p. 240].

\subsection{Three Point-Line Constraints}

Finding the number of positions that a rigid body can adopt so that three of its points lie on three particular lines is now straightforward. All that is needed is to intersect three $\mathbb{P}^{1} \times \mathbb{P}^{3}$ Segre varieties. This is best done in one of the Segre varieties.

Corollary 9 In general, there are 8 ways that a rigid-body can be positioned so that 3 given points of the body lie on 3 particular lines, each point lying on its own particular line.

Proof As shown above, the intersection of two of these Segre varieties is a surface with homology $2 \alpha_{1} \beta_{1}+2 \alpha_{0} \beta_{2}$. The intersection of two of these surfaces, that is the intersection of two Segre varieties with a third, will have homology,

$$
\left(2 \alpha_{1} \beta_{1}+2 \alpha_{0} \beta_{2}\right)^{2}=8 \alpha_{0} \beta_{0} .
$$

That is, eight solutions can be expected in general.

This result is consistent with those given by Zsombor-Murray and Gfrerrer [11] who consider the 'spatial double triangular mechanism'. 


\section{Intersection of Point-Plane Constraints}

In this section the intersection of several point-plane constraints is considered. This is the problem considered by Wampler [10] where the degrees of these intersections are computed using numerical techniques. Wampler uses several different models for the group of rigid displacements and so it is difficult, in general, to compare results.

Here the intersection with the Study quadric is considered so the homology class of the constraint as a subvariety of the Study quadric is often the appropriate invariant to find. The homology of the non-singular quadrics was found by Ehresmann [2]. The restriction of this general result to the case of the Study quadric can be found in [7, §11.5]. Essentially, the homology is generated by a single class in each dimension except dimension 3 . The homology of a 3 dimensional subvariety of the Study quadric is an integer sum of two classes $\sigma_{A}$ and $\sigma_{B}$. The two sets of 3-plane generators of the six-dimensional quadric are know as $A$-planes and $B$-planes, the class of an $A$-plane is $\sigma_{A}$ and similarly $\sigma_{B}$ is the class of a single $B$-plane. The generic intersections of these generator planes leads to the intersection formulas $\sigma_{A} \cap \sigma_{B}=\sigma_{0}$ the class of a point, and $\sigma_{A} \cap \sigma_{A}=\sigma_{B} \cap \sigma_{B}=0$. The intersection of the Study quadric with a hyperplane in $\mathbb{P}^{7}$ gives a five dimensional subvariety with class $\sigma_{5}$. Similarly, the intersection with a 5-plane give a subvariety with class $\sigma_{4}$. A tangent 4plane intersect the Study quadric in an $A$-plane and a $B$-plane, more generally a 4-plane will intersect the Study quadric in a subvariety with class $\sigma_{A}+\sigma_{B}$. It is not hard to see that if a 4 or 5-dimensional subvariety has class $d \sigma_{4}$ or $e \sigma_{5}$ then as a subvariety of $\mathbb{P}^{7}$ the space will have degree $2 d$ and $2 e$ respectively. Also a 3 -dimensional subvariety with homology class $d_{a} \sigma_{A}+d_{b} \sigma_{B}$ will have degree $d_{a}+d_{b}$ as a subvariety of $\mathbb{P}^{7}$. In dimensions less that 3 , the homology of the Study quadric and the degree in $\mathbb{P}^{7}$ are effectively the same, for example a curve in the Study quadric with class $2 \sigma_{1}$ is simply a conic in $\mathbb{P}^{7}$.

\subsection{One Point-Plane Constraint}

The homology class of a single point-plane constraint, as a subvariety of the Study quadric is simply $2 \sigma_{5}$. This is because the subvariety consists of the intersection of the Study quadric with a quadric hypersurface.

Proposition 7 The set of group elements which maintain the incidence of a point with a plane lie on a variety which is the projection of the Segre variety $\mathbb{P}^{2} \times \mathbb{P}^{3}$ in $\mathbb{P}^{11}$ to $\mathbb{P}^{7}$.

Proof This set of group elements can also be easily parameterised as a product of an arbitrary rotation about the point followed by a translation in the plane. To keeps computations simple let us assume that the point under consideration in initially located at the origin and the plane in question is the $x y$-plane. The possible group elements satisfying the constraint can then be written as the dual quaternion,

$$
a+\varepsilon c=\left(\lambda_{0}+\varepsilon\left(\lambda_{1} i+\lambda_{2} j\right)\right)\left(\mu_{0}+\mu_{1} i+\mu_{2} j+\mu_{3} k\right)
$$

where $\lambda_{i}$ and $\mu_{j}$ are real parameters. Expanding the product gives,

$$
\begin{aligned}
& a=\lambda_{0} \mu_{0}+\lambda_{0} \mu_{1} i+\lambda_{0} \mu_{2} j+\lambda_{0} \mu_{3} k \\
& \begin{array}{r}
c=-\left(\lambda_{1} \mu_{1}+\lambda_{2} \mu_{2}\right)+\left(\lambda_{1} \mu_{0}+\lambda_{2} \mu_{3}\right) i+ \\
\quad\left(\lambda_{2} \mu_{0}-\lambda_{1} \mu_{3}\right) j+\left(\lambda_{1} \mu_{2}-\lambda_{2} \mu_{1}\right) k .
\end{array}
\end{aligned}
$$


Notice that the coefficients of the quaternions $a$ and $c$ are all separately linear in the $\lambda_{i}$ and $\mu_{j}$ parameters. It is straightforward to check that such group elements do indeed satisfy the constraint and of course the Study quadric. Now consider the parameters $\left(\lambda_{0}: \lambda_{1}: \lambda_{2}\right)$ and $\left(\mu_{0}: \mu_{1}: \mu_{2}: \mu_{3}\right)$ as homogeneous coordinates for a $\mathbb{P}^{2}$ and a $\mathbb{P}^{3}$ respectively. The standard Segre embedding of $\mathbb{P}^{2} \times \mathbb{P}^{3}$ in $\mathbb{P}^{11}$ is given by assigning each of the 12 homogeneous coordinates in $\mathbb{P}^{11}$ a term $\lambda_{i} \mu_{j}$ where $0 \leq i \leq 2$ and $0 \leq j \leq 3$, see [3, p. 25]. Clearly the eight linear functions of the $\lambda_{i} \mu_{j}$ cannot span all 12 possible combinations. There must be a linear space of $4 \lambda_{i} \mu_{j}$ s not appearing in the in the parameterisation. Geometrically this can be thought of as a projection to a $\mathbb{P}^{7}$ with a $\mathbb{P}^{3}$ as centre.

\subsection{Two Point-Plane Constraints}

Consider the set of rigid displacements which preserve the incidence of a pair of points with a pair of planes. Note that the planes will, in general, intersect in a line. Consider a line in the first plane perpendicular to the line of intersection. Let us call this line $L$. Now the possible rigid displacements can be split into a displacement which preserves the incidence of the first point with the line $L$ and the second point with the second plane, followed by a translation parallel to the intersection line of the two planes. As shown above the set of displacements which constrain a point to a line form a Segre variety $\mathbb{P}^{1} \times \mathbb{P}^{3}$. The displacements which also constrain a second point to lie on a plane will form a 3-dimensional subvariety of the Segre variety, call this subvariety $X$ say. It is useful to consider the geometry of $X$ first.

Lemma 1 The the 3-dimensional variety $X$ has degree 7.

Proof The variety $X$ is the intersection of the Segre variety $\mathbb{P}^{1} \times \mathbb{P}^{3}$ with a quadratic constraint $\mathrm{Q}_{\pi}(p, n)$. Coordinates can be chosen so that the first point is initially located at the origin, the first plane coincides with the $x y$-plane and the line of intersection between the two planes is parallel to the $y$-axis. In these coordinates the line $L$ will lie along the $x$-axis, so the Segre variety $\mathbb{P}^{1} \times \mathbb{P}^{3}$ can be parameterised as in section 5.1 above, as $g=a+\varepsilon c$ with,

$$
\begin{aligned}
& a=\lambda_{0} \mu_{0}+\lambda_{0} \mu_{1} i+\lambda_{0} \mu_{2} j+\lambda_{0} \mu_{3} k, \\
& c=-\lambda_{1} \mu_{1}+\lambda_{1} \mu_{0} i-\lambda_{1} \mu_{3} j+\lambda_{1} \mu_{2} k .
\end{aligned}
$$

Now these can be substituted into the matrix equation for $\mathrm{Q}_{\pi}(p, n)$ as in section 3.1 above. The result will be a bi-homogeneous polynomial in the parameters, $\lambda_{i}$ and $\mu_{j}$. However, the structure of the matrix for $\mathrm{Q}_{\pi}(p, n)$, the zero submatrix in the bottom left-hand corner, means that $\lambda_{0}$ will be a common factor in all terms of the equation. Hence, this factor may be cancelled and the result will be a polynomial which is homogeneous of degree 1 in the $\lambda_{i}$ variables and homogeneous of degree 2 in the $\mu_{j}$ variables. Hence, as a subvariety of the Segre variety, $X$ will have homology class $\alpha_{0} \beta_{3}+2 \alpha_{1} \beta_{2}$. As a subvariety of $\mathbb{P}^{7}$, the degree of the variety $X$ will therefore be,

$$
\left(\begin{array}{l}
3 \\
0
\end{array}\right)+2\left(\begin{array}{l}
3 \\
1
\end{array}\right)=1+2 \times 3=7
$$

see $[3, \S 19.1]$. 
Remark 6 The homology class of $X$ as a subvariety of the Study quadric can be found as follows. Recall that the Segre variety $\mathbb{P}^{1} \times \mathbb{P}^{3}$ has degree 4 , hence as a subvariety of the Study quadric its homology class must be $2 \sigma_{4}$. Intersecting with the quadric $Q_{\pi}(p, n)$ with homology class $2 \sigma_{5}$ gives a variety with class,

$$
2 \sigma_{4} \cap 2 \sigma_{5}=4 \sigma_{A}+4 \sigma_{B} .
$$

However, notice that the Segre variety and the quadratic constraint both contain the 3-plane $A_{\infty}$ of ideal points in the Study quadric. This 3-plane must be a component of the intersection, but does not correspond to any physical group elements. Deleting this component leaves a variety with class $3 \sigma_{A}+4 \sigma_{B}$. The degree of this variety, as a subvariety of $\mathbb{P}^{7}$ will be $3+4=7$ and hence we can identify this as the subvariety $X$.

Remark 7 It is also possible to find a parameterisation for $X$. With the same coordinates as above, imagine rotating the rigid-body about the first point at the origin. This will move the second point off its plane in general. The incidence between the second point and plane can be regained by a suitable translation along $L$. Suppose the original rotation is given by a quaternion $\left(\mu_{0}+\mu_{1} i+\mu_{2} j+\mu_{3} k\right)$ with arbitrary parameters $\mu_{i}$, the corresponding $3 \times 3$ rotation matrix will be,

$$
R=\frac{1}{\Delta}\left(\begin{array}{ccc}
\mu_{0}^{2}+\mu_{1}^{2}-\mu_{2}^{2}-\mu_{3}^{2} & 2\left(\mu_{1} \mu_{2}-\mu_{0} \mu_{3}\right) & 2\left(\mu_{1} \mu_{3}+\mu_{0} \mu_{2}\right) \\
2\left(\mu_{1} \mu_{2}+\mu_{0} \mu_{3}\right) & \mu_{0}^{2}-\mu_{1}^{2}+\mu_{2}^{2}-\mu_{3}^{2} & 2\left(\mu_{2} \mu_{3}-\mu_{0} \mu_{1}\right) \\
2\left(\mu_{1} \mu_{3}-\mu_{0} \mu_{2}\right) & 2\left(\mu_{2} \mu_{3}+\mu_{0} \mu_{1}\right) & \mu_{0}^{2}-\mu_{1}^{2}-\mu_{2}^{2}+\mu_{3}^{2}
\end{array}\right)
$$

where $\Delta=\mu_{0}^{2}+\mu_{1}^{2}+\mu_{2}^{2}+\mu_{3}^{2}$, see for example [7, §2.2.2]. If the second point is $\mathbf{p}$ and the normal to the second plane is $\mathbf{n}$ then the required translation $\mathbf{t}=t_{x} i$, will satisfy,

$$
\mathbf{n}^{T} R \mathbf{p}+\mathbf{n}^{T} \mathbf{t}=\mathbf{n}^{T} \mathbf{p} .
$$

So that,

$$
n_{x} t_{x}=\mathbf{n}^{T}(I-R) \mathbf{p}
$$

with $n_{x}$ the $x$-component of the normal vector $\mathbf{n}$. Now it is convenient to write the rotation matrix as $R=\frac{1}{\Delta} \tilde{R}$, where $\tilde{R}$ is the matrix displayed above with components that are quadratic functions of the $\mu_{i}$ s. The dual quaternion representing this translation can then be written, $\Delta+\varepsilon \Gamma i$, where,

$$
\Gamma=\frac{1}{2 n_{x}} \mathbf{n}^{T}(\Delta I-\tilde{R}) \mathbf{p} .
$$

Combining this with the arbitrary rotation about the origin gives a dual quaternion with components,

$$
\begin{aligned}
& a=\Delta \mu_{0}+\Delta \mu_{1} i+\Delta \mu_{2} j+\Delta \mu_{3} k \\
& c=-\Gamma \mu_{1}+\Gamma \mu_{0} i-\Gamma \mu_{3} j+\Gamma \mu_{2} k
\end{aligned}
$$

Notice that each component of this dual quaternion is a cubic function of the parameters $\mu_{i}$, since both $\Gamma$ and $\Delta$ are quadratic functions of the parameters.

Proposition 8 The variety of group elements which preserve the incidence of two points with two planes has degree 8.

Proof It is easy to see that such a variety must have homology class $2 \sigma_{5} \cap 2 \sigma_{5}=4 \sigma_{4}$ and hence as a subvariety of $\mathbb{P}^{7}$ will have degree 8 . 
Remark 8 As described above, the required group elements can be split into an element from the subvariety $X$ followed by an arbitrary translation in the direction of the line of intersection between the two planes. With coordinates as above, this can be written as a dual quaternion,

$$
g=\left(v_{0}+\varepsilon v_{1} j\right)\left(\left(\Delta \mu_{0}+\Delta \mu_{1} i+\Delta \mu_{2} j+\Delta \mu_{3} k\right)+\varepsilon\left(-\Gamma \mu_{1}+\Gamma \mu_{0} i-\Gamma \mu_{3} j+\Gamma \mu_{2} k\right)\right),
$$

where $v_{0}$ and $v_{1}$ are arbitrary constants (not both zero). When $v_{1}=0$ the variety $X$ is recovered, but when $v_{0}=0$ the result is a point on the 3-plane $A_{\infty}$. That is a point of the form $0+\varepsilon\left(\mu_{0}+\mu_{1} i+\mu_{2} j+\mu_{3} k\right)$, ignoring common factors. From this it is easy to see that the intersection of two point-plane constraints is the join of corresponding points on the varieties $X$ and $A_{\infty}$.

\subsection{Three Point-Plane Constraints}

The homology computation for three point-plane constraints is simply,

$$
2 \sigma_{4} \cap 2 \sigma_{4} \cap 2 \sigma_{4}=8 \sigma_{A}+8 \sigma_{B}
$$

However, the 3-plane $A_{\infty}$ is common to all point-plane quadrics. So this 3-plane must be a component of the three dimensional intersection.

Lemma 2 The multiplicity of the component $A_{\infty}$ in the intersection of three point-plane constraints is one.

Proof To show this it is enough to show that the variety $A_{\infty}$ is non-singular. The partial derivatives of a quadric of the form $\mathbf{g}^{T} \mathrm{Q}_{\pi}(n, p) \mathbf{g}=0$ can be written in vector form as, $\mathrm{Q}_{\pi}(p, n)$ g. That is, the partial derivative with respect to $a_{0}$ is the first component of the vector, the partial derivative with respect to $a_{1}$ is the second component and so forth. Hence the singularities of the variety occur when the vectors,

$$
\mathrm{Q}_{\pi}\left(p_{1}, n_{1}\right) \mathbf{g}, \quad \mathrm{Q}_{\pi}\left(p_{2}, n_{2}\right) \mathbf{g}, \quad \mathrm{Q}_{\pi}\left(p_{3}, n_{3}\right) \mathbf{g}, \quad \mathrm{Q}_{0} \mathbf{g},
$$

are linearly dependant. The last vector here comes from the partial derivatives of the Study quadric. On the 3-plane $A_{\infty}$ the condition for singularity becomes simply,

$$
\operatorname{det}\left(\mathscr{N}_{1} \mathbf{c}\left|\mathscr{N}_{2} \mathbf{c}\right| \mathscr{N}_{3} \mathbf{c} \mid \mathbf{c}\right)=0
$$

Where $\mathbf{c}=\left(c_{0}, c_{1}, c_{2}, c_{3}\right)^{T}$ and $\mathscr{N}_{i}$ is the top right-hand block of the matrix for $\mathrm{Q}_{\pi}\left(p_{i}, n_{i}\right)$. The matrix in this equation is not identically zero so $A_{\infty}$ is in general non-singular and hence can only have multiplicity 1 in the variety.

Proposition 9 The set of group elements which preserve the incidence of three points on a rigid body with three planes lie on a variety of degree 15.

Proof From above, the intersection of three point-plane constraints will have homology class $8 \sigma_{A}+8 \sigma_{B}$. Deleting a single copy of the component $A_{\infty}$ leaves a variety with homology class $7 \sigma_{A}+8 \sigma_{B}$. So the degree of this variety as a subvariety of $\mathbb{P}^{7}$ is $7+8=15$.

This result agrees with that given by Wampler [10]. 
Remark 9 This variety can also be parameterised as in the previous section. Once again let $L$ be the line in the first plane perpendicular to the line of intersection between the first and second planes. Now rotate the rigid body about the first point, and then translate along $L$ until the second point regains contact with the second plane. Finally, translate the body parallel to the intersection of the first and second planes until the third point regains contact with the third plane. With the coordinates introduced above, that is with the first point initially at the origin, the first plane the $x y$-plane and the line $L$ along the $x$-axis, the third point can be labelled $\mathbf{q}$ and the normal to the third plane $\mathbf{m}$. Using $t_{y}$ to represent the translation parallel to the intersection of the first and second planes the third point must satisfy,

$$
\mathbf{m}^{T}\left(R \mathbf{q}+t_{x} i+t_{y} j\right)=\mathbf{m}^{T} \mathbf{q} .
$$

Hence,

$$
t_{y}=\frac{1}{m_{y}}\left(\mathbf{m}^{T}(I-R) \mathbf{q}-m_{x} t_{x}\right) .
$$

Writing the dual quaternion representing the translation as $\Delta+\varepsilon \Xi j$ with $t_{x}=2 \Gamma / \Delta$ as in the previous section, it is clear that,

$$
\Xi=\frac{\Delta}{2} t_{y}=\frac{1}{2 m_{y}}\left(\mathbf{m}^{T}(\Delta I-\tilde{R}) \mathbf{q}-2 m_{x} \Gamma\right) .
$$

Note that this quantity is again quadratic in the parameters $\mu_{i}$, because $\Delta, \tilde{R}$ and $\Gamma$ are quadratic in the parameters. So combining the rotation, translation along $L$ with the translation parallel to the intersection of the first and second planes gives,

$$
g=(\Delta+\varepsilon \Xi j)(\Delta+\varepsilon \Gamma i) \mu,
$$

where $\mu=\mu_{0}+\mu_{1} i+\mu_{2} j+\mu_{3} k$ is the quaternion representing the rotation about the first point. Cancelling the common factor $\Delta$ the required group elements can be parameterised as

$$
g=(a+\varepsilon c)=\Delta \mu+\varepsilon(\Gamma i \mu+\Xi j \mu) .
$$

Notice that this variety is again parameterised by cubic functions of the $\mu_{i} \mathrm{~s}$.

Remark 10 This variety intersects the 3-plane $A_{\infty}$ when $\Delta=\mu \mu^{-}=\mu_{0}^{2}+\mu_{1}^{2}+\mu_{2}^{2}+\mu_{3}^{2}=0$. When this is the case, the pure dual part of the group element $c$, satisfies the relation $c c^{-}=$ $\left(\Xi^{2}+\Gamma^{2}\right) \mu \mu^{-}=0$. Since $\mu \neq 0$ these are the only solutions and we may conclude that the intersection of the variety with the 3-plane $A_{\infty}$ is the imaginary 2-dimensional sphere, $a_{0}=a_{1}=a_{2}=a_{3}=0, c_{0}^{2}+c_{1}^{2}+c_{2}^{2}+c_{3}^{3}=0$. This 2 -sphere will be referred to as $S_{\infty}$ from now on.

\subsection{Schönflies Motion Constrained by Three Point-Plane Constraints}

A Schönflies motion is a motion that is restricted to a certain 4-dimensional subgroup of the group of rigid-body displacements. These Schönflies subgroups consist of a rotation about an axis together with any translation. The subgroup is sometimes described as the possible motion of a waiter's tray, the plane of the tray must be kept horizontal, but any translation or rotation about a vertical axis is allowed. As a subvariety of the Study quadric such a subgroup is represented by the intersection of the Study quadric with a 5-plane, see [8]. If $\left(\alpha_{1}, \beta_{1}, \gamma_{1}\right)^{T}$ and $\left(\alpha_{2}, \beta_{2}, \gamma_{2}\right)^{T}$ are independent normal vectors perpendicular to the allowed 
rotation axis of the Schönflies motion, then the linear equations in $\mathbb{P}^{7}$ satisfied by the motion are,

$$
\alpha_{1} a_{1}+\beta_{1} a_{2}+\gamma_{1} a_{3}=0, \quad \text { and } \quad \alpha_{2} a_{1}+\beta_{2} a_{2}+\gamma_{2} a_{3}=0 .
$$

Another way of putting this is that the possible rotations can be parameterised by the quaternion $a=\mu_{0}+\mu_{1} \mathbf{v}$ where $\mathbf{v}$ is a vector in the direction of the rotation axis.

Proposition 10 The intersection of the variety formed by three point-plane constraints with a 5-plane which determines a Schönflies motion results in a Darboux motion.

Before proving this, note that Darboux motions are characterised by the fact that the trajectory of any point in space moving under such a motion remain on a plane, actually they follow conic trajectories. It is well known that Darboux motions are represented by certain twisted cubic curves in the Study quadric, [1, Ch. 9 §3].

Proof Substitute the parameterisation of the constraint variety (4) from the previous section into the two linear equations above. The result gives two possibilities, first $\Delta=\mu_{0}^{2}+\mu_{1}^{2}+$ $\mu_{2}^{2}+\mu_{3}^{2}=0$, this is the imaginary 2-sphere of ideal points $S_{\infty}$. The second possibility is the curve given by, $\mu=\mu_{0}+\mu_{1} \mathbf{v}$. Substituting this solution into (4) gives a curve in the Study quadric where the 8 coordinates, $a_{i}, c_{j}$ are given by cubic functions of the parameters $\mu_{0}$ and $\mu_{1}$. Such a curve is, in general, a twisted cubic curve.

To show that this curve corresponds to a Darboux motion consider an arbitrary point given by the dual quaternion, $p_{0}+\varepsilon \mathbf{p}$. As shown is section 2 above, the action of the group of such a point is given by,

$$
(a+\varepsilon c)\left(p_{0}+\varepsilon \mathbf{p}\right)\left(a^{-}-\varepsilon c^{-}\right)=p_{0}^{\prime}+\varepsilon \mathbf{p}^{\prime} .
$$

Substituting into this equation from (4) above gives,

$$
\begin{array}{r}
\Delta \mu+\varepsilon(\Gamma i \mu+\Xi j \mu)\left(p_{0}+\varepsilon \mathbf{p}\right) \Delta \mu^{-}+\varepsilon\left(\Gamma \mu^{-} i+\Xi \mu^{-} j\right)= \\
\Delta^{3} p_{0}+\varepsilon \Delta^{2}\left(\mu \mathbf{p} \mu^{-}+2 p_{0}(\Gamma i+\Xi j)\right),
\end{array}
$$

were the relation $\Delta=\mu \mu^{-}$has been used to simplify the result. Cancelling the common factor $\Delta^{2}$ gives,

$$
p_{0}^{\prime}+\varepsilon \mathbf{p}^{\prime}=\Delta p_{0}+\varepsilon\left(\mu \mathbf{p} \mu^{-}+2 p_{0}(\Gamma i+\Xi j)\right) .
$$

Finally substituting $\mu=\mu_{0}+\mu_{1} \mathbf{v}$, gives a curve in $\mathbb{P}^{3}$ in which all the coordinates are quadratic functions of $\mu_{0}$ and $\mu_{1}$, since $\Delta, \Gamma$ and $\Xi$ are quadratic functions of these parameters.

This result appears in Zsombor-Murray and Gfrerrer [11]. It would be a little easier to show given a characterisation of the Darboux motion as a twisted cubic curve meeting $S_{\infty}$ in two points.

\subsection{The Tripod mechanism}

In this section the tripod mechanism studied by Wampler [10], is considered. Essentially the mechanism consists of three points, at the vertices of an equilateral triangle, constrained to lie on three symmetrically disposed planes. The three planes all meet in a single line and are separated by angles of $2 \pi / 3$ radians. 
Proposition 11 The possible displacements of a general tripod mechanism lie in a degree 7 variety.

Proof The quadrics defining the three point-plane constraints can be taken as, $\mathrm{Q}_{\pi}(n, p)$, $G^{T} \mathrm{Q}_{\pi}(n, p) G$ and $\left(G^{2}\right)^{T} \mathrm{Q}_{\pi}(n, p) G^{2}$, where $G$ is the $8 \times 8$ matrix representing a rotation of $2 \pi / 3$ radians about the common line of the three planes. If we take $p$ to be a point on the $x$-axis and $n$ to be in the $y$-direction then the common line can be taken as the $z$-axis and $G$ as a rotation of $2 \pi / 3$ radians about the $z$-axis. After some straightforward computations the quadric corresponding to the sum, $\mathrm{Q}_{\pi}(n, p)+G^{T} \mathrm{Q}_{\pi}(n, p) G+\left(G^{2}\right)^{T} \mathrm{Q}_{\pi}(n, p) G^{2}$, is found to be $a_{0} a_{3}=0$. That is, the quadric degenerates to a pair of hyperplanes, $a_{0}=0$ and $a_{3}=0$. The intersection of two point-plane constraints with a hyperplane will give a 3-dimensional variety in the Study quadric with homology class $4 \sigma_{A}+4 \sigma_{B}$. Both hyperplanes contain the 3 -plane of ideal elements $A_{\infty}$. Deleting this component gives a subvariety of class $3 \sigma_{A}+4 \sigma_{B}$, in each case. The degree of such a variety in $\mathbb{P}^{7}$ is just $3+4=7$.

The hyperplane $a_{0}=0$ consist of the rigid displacements with rotation angle $\pi$, so this component is not usually considered relevant since a practical machine would find it difficult to perform such a displacement. On the other hand, the hyperplane $a_{3}=0$ contains the rigid displacements that have no rotation about the $z$-axis; the common line in the three planes.

Again these results agree with the numerical computations in [10]. However, the results derived here are a little more detailed. For example, suppose that we attached a chain of three revolute joints between the rigid body containing the three points of the tripod mechanism and the ground.

Proposition 12 The number of possible assembly configurations of this mechanism will be, in general, 20.

Proof The variety of rigid displacements allowed by the three-revolute chain is known to have class $2 \sigma_{A}+4 \sigma_{B}$, see [7, p.263]. Hence the number of assembly configurations for this composite mechanism can be estimated to be,

$$
\left(3 \sigma_{A}+4 \sigma_{B}\right) \cap\left(2 \sigma_{A}+4 \sigma_{B}\right)=3 \times 4+4 \times 2=20 .
$$

\subsection{Six Point-Plane Constraints}

Here we look at the intersection of six point-plane constraints with the Study quadric. Each point-plane constraint is a quadrics of the form, $\mathbf{g}^{T} \mathrm{Q}_{\pi}(\mathbf{p}, \mathbf{n}) \mathbf{g}=0$, where the form of $Q_{\pi}(\mathbf{p}, \mathbf{n})$ is given in equation (3) above. Now suppose the normal vectors for the six planes are $\mathbf{n}_{1}, \mathbf{n}_{2}, \ldots, \mathbf{n}_{6}$, only three of these can be linearly independent, so in general there will be relations,

$$
\begin{aligned}
& \phi_{1} \mathbf{n}_{1}+\phi_{2} \mathbf{n}_{2}+\phi_{3} \mathbf{n}_{3}+\phi_{4} \mathbf{n}_{4}=\mathbf{0}, \\
& \chi_{1} \mathbf{n}_{1}+\chi_{2} \mathbf{n}_{2}+\chi_{3} \mathbf{n}_{3}+\chi_{5} \mathbf{n}_{5}=\mathbf{0} \\
& \psi_{1} \mathbf{n}_{1}+\psi_{2} \mathbf{n}_{2}+\psi_{3} \mathbf{n}_{3}+\psi_{6} \mathbf{n}_{6}=\mathbf{0}
\end{aligned}
$$

with $\phi_{i}, \chi_{i}$ and $\psi_{i}$ some constants that are not all zero. If the quadrics are substituted into these equations the resulting quadrics will have the form,

$$
\phi_{1} \mathrm{Q}_{\pi}\left(\mathbf{p}_{1}, \mathbf{n}_{1}\right)+\phi_{2} \mathrm{Q}_{\pi}\left(\mathbf{p}_{2}, \mathbf{n}_{2}\right)+\phi_{3} \mathrm{Q}_{\pi}\left(\mathbf{p}_{3}, \mathbf{n}_{3}\right)+\phi_{4} \mathrm{Q}_{\pi}\left(\mathbf{p}_{4}, \mathbf{n}_{4}\right)=\left(\begin{array}{ll}
\Phi & 0 \\
0 & 0
\end{array}\right),
$$


where

$$
\Phi=\phi_{1} \mathscr{W}_{1}+\phi_{2} \mathscr{W}_{2}+\phi_{3} \mathscr{W}_{3}+\phi_{4} \mathscr{W}_{4}
$$

is some symmetric $4 \times 4$ matrix. With similar results for the other two equations. The quadric defined by the $8 \times 8$ symmetric matrices represent cones (projectively equivalent to a cylinder) in $\mathbb{P}^{7}$.

Proposition 13 In general there will be 8 group elements which satisfy six point-plane constraints.

Proof The intersection of the six original quadrics is clearly the same as the intersection of these cones with three of the original quadrics. The intersection of the three cones with the $A$-plane $c_{0}=c_{1}=c_{2}=c_{3}=0$ of rotations about the origin is, in general, 8 distinct points. Let us call this $A$-plane $A_{0}$ for short. For each of these points a point in the intersection of all the quadrics can be found. To do this suppose that the coordinates in $A_{0}$ are given by $\overline{\mathbf{a}}=\left(\bar{a}_{0}, \bar{a}_{1}, \bar{a}_{2}, \bar{a}_{2}\right)^{T}$. Now substitute the values found for intersection of the three cones $\overline{\mathbf{a}}$ into the first three quadrics,

$$
\overline{\mathbf{a}}^{T} \mathscr{W}_{i} \overline{\mathbf{a}}+2 \overline{\mathbf{a}}^{T} \mathscr{N}_{i} \mathbf{c}=0, \quad i=1,2,3
$$

and the Study quadric, $\overline{\mathbf{a}}^{T} \mathbf{c}=0$. For any of the 8 points $\overline{\mathbf{a}}$ is known, so these are linear equations for $\mathbf{c}$. The four equations for the 4 unknowns can be written in a matrix form as,

$$
M \mathbf{c}=\mathbf{b}
$$

where $M$ is the matrix with rows given by,

$$
M=-2\left(\begin{array}{c}
\overline{\mathbf{a}}^{T} \mathscr{N}_{1} \\
\overline{\mathbf{a}}^{T} \mathscr{N}_{2} \\
\overline{\mathbf{a}}^{T} \mathscr{N}_{3} \\
\overline{\mathbf{a}}^{T}
\end{array}\right) \quad \text { and } \quad \mathbf{b}=\left(\begin{array}{c}
\overline{\mathbf{a}}^{T} \mathscr{W}_{1} \overline{\mathbf{a}} \\
\overline{\mathbf{a}}^{T} \mathscr{W}_{2} \overline{\mathbf{a}} \\
\overline{\mathbf{a}}^{T} \mathscr{W}_{3} \overline{\mathbf{a}} \\
0
\end{array}\right)
$$

Now since $\mathbf{a}$ and $\mathbf{c}$ are homogeneous coordinates in a $\mathbb{P}^{7}$ we only need to solve these equations up to an overall multiplicative factor, the solution can be written,

$$
\mathbf{a}=\operatorname{det}(M) \overline{\mathbf{a}}, \quad \text { and } \quad \mathbf{c}=\operatorname{Adj}(M) \mathbf{b}
$$

where $\operatorname{Adj}(M)$ represents the adjugate matrix of $M$. In general, we obtain 8 distinct solutions corresponding to the 8 intersection of the three cones with $A_{0}$.

Remark 11 Notice that the intersection of the six quadrics with the Study quadric may contain additional solutions and even whole components of positive dimension, but these features can only be located in the ideal plane, $A_{\infty}$. Since these solutions will not be physical group elements they are not really of any interest. Note also that the solution count here agrees with the results of Wampler [10]. 


\subsection{Five Point-Plane Constraints}

We may treat the case of five point-plane constraints in a very similar fashion to the 6 constraints considered above. In this case there are only two quadratic cones and their intersection with $A_{0}$ is, in general, an elliptic quartic curve. This process of elimination is of course geometrically a projection, moreover a linear projection with $A_{\infty}$ as the centre of the projection. The pre-image of the projection can be recovered as above by setting,

$$
\mathbf{a}=\operatorname{det}(M) \overline{\mathbf{a}}, \quad \text { and } \quad \mathbf{c}=\operatorname{Adj}(M) \mathbf{b} .
$$

Note that this will give a point on $A_{\infty}$, the centre of the projection, exactly when $\operatorname{det}(M)=0$.

Lemma 3 The determinant $\operatorname{det}(M)=0$ if and only if $\overline{\mathbf{a}}^{T} \overline{\mathbf{a}}=0$.

Proof Notice that,

$$
M \overline{\mathbf{a}}=\left(\begin{array}{c}
0 \\
0 \\
0 \\
\overline{\mathbf{a}}^{T} \overline{\mathbf{a}}
\end{array}\right),
$$

since the $\mathscr{N}_{i}$ matrices are anti-symmetric. Moreover it is easy to see that a multiple of the vector $\overline{\mathbf{a}}$ is the only non-zero vector which is annulled by the first three columns of $M$. Hence when $\overline{\mathbf{a}}^{T} \overline{\mathbf{a}}=0$ the matrix has a non-trivial kernel.

Proposition 14 The possible motions of a rigid-body subject to five point-plane constraints lie on an elliptic curve of degree 12.

Proof The equation $\operatorname{det}(M)=0$ defines a complex quadric surface $\overline{\mathbf{a}}^{T} \overline{\mathbf{a}}=0$, in $A_{0}$. This surface will in general meet the elliptic quartic curve found above in $4 \times 2=8$ points. Hence there are in general 8 points where the curve in $\mathbb{P}^{7}$ meets the centre of the projection. When this curve is projected to $A_{0}$ its degree is reduced by one for each intersection with the centre of projection. Hence, we can infer that the degree of the curve which projects to the elliptic quartic in $A_{0}$ must have degree $4+8=12$. Moreover, since it is known that the genus of a curve is invariant under projections we may also conclude that this curve is elliptic.

Again this result for the degree of this variety agree with the results of Wampler [10].

\subsection{Four Constraints}

The result in this section differs from that found by Wampler [10], although the comment made above on the difficulty of making direct comparisons of results should be borne in mind. The result given here is however, consistent with those found in the previous section and in section 5.2 above.

Proposition 15 The set of group elements which preserve the incidence of four points with four planes lie on a surface of degree 6.

Proof For 4 point-plane constraints there is just one quadratic cone in the linear system of quadrics. The intersection of this cone with the 3-plane $A_{0}$ is, in general, a non-singular 2dimensional quadric. The intersection of this quadric with the imaginary quadric $\operatorname{det}(M)=$ $\overline{\mathbf{a}}^{T} \overline{\mathbf{a}}=0$ will be an elliptic quartic curve in general. Now suppose we intersect these varieties 
with a hyperplane in $\mathbb{P}^{7}$. In $A_{0}$ the intersection with the quadratic cone will be a conic curve and the intersection with the quartic curve will be 4 points. Using the same argument as in the previous section we may conclude that the intersection of the 4 point-plane constraints with the hypersurface is a curve with degree $2+4=6$. So a general hyperplane section of this surface is a degree 6 curve, thus the degree of the surface is also 6.

Remark 12 The surface is birationally equivalent to $\mathbb{P}^{1} \times \mathbb{P}^{1}$. The intersection of the cone from the linear system of point-plane constraints with $A_{0}$ gives a 2-dimensional quadric. This surface is well known to be isomorphic to the Segre variety $\mathbb{P}^{1} \times \mathbb{P}^{1}$, so this variety can be parametrised by linear functions of the products $\lambda_{i} \mu_{j}$ where $i$ and $j$ are 0 or 1 . That is, the coordinates of $\overline{\mathbf{a}}$ are linear functions of the $\lambda_{i} \mu_{j}$. As above the surface in $\mathbb{P}^{7}$ can be reconstructed as,

$$
\mathbf{a}=\operatorname{det}(M) \overline{\mathbf{a}}, \quad \text { and } \quad \mathbf{c}=\operatorname{Adj}(M) \mathbf{b} .
$$

This gives a rational map from $\mathbb{P}^{1} \times \mathbb{P}^{1}$ to the surface which inverts the projection to $A_{0}$. Notice here that the elements of the matrix $M$ are linear functions of the $\lambda_{i} \mu_{j}$ while the coordinates of the vector $\mathbf{b}$ are quadratic functions of $\lambda_{i} \mu_{j}$.

\section{Conclusions}

This work has looked at general results for the intersections of point-plane constraints on rigid body displacements. There are however many special cases where these results will fail. For example, the Darboux motion mentioned in section 6.4 above is characterised by the fact that under this motion all point of space move on non-parallel planes. Hence there must be choices of 6 point-plane constraints whose intersection is a curve rather than eight points. This also applies to the infinitesimal kinematics studied in section 4 . For a Darboux motion the zero torsion cubic must degenerate to all of space. The study of the possible degeneracies, both for finite and infinitesimal kinematics, and the conditions under which they occur is important future work.

Another area of future work which follows from the above is the investigation of intersections of the point-sphere constraints. This has particular relevance to the kinematics of a parallel robot manipulator known as the Gough-Stewart platform. This machine is known to have, in general, 40 possible solutions for the position of the platform given particular lengths for the legs. That is the intersection of 6 point-sphere constraints should yield 40 solutions, see [9], [4] and [5], for example.

Finally, it is hoped that the case has been made for the simple techniques from Algebraic Geometry that have been used here. Although more sophisticated techniques will be able to take us further these simple methods still have much to offer.

\section{References}

1. Bottema, O. and Roth, B., Theoretical Kinematics, Dover Publications, New York, (1990).

2. Ehresmann, C., Sur la topologie de certaines espaces homogènes, Ann. of Math., 396-443, (1934).

3. Harris, J., Algebraic Geometry a first course, Springer Verlag, New York, (1992).

4. Husty, M. L., An algorithm for solving the direct kinematics of general Stewart-Gough platforms. Mechanism and Machine Theory, 31(4):365-380, (1996).

5. Mourrain, B., Enumeration problems in Geometry, Robotics and Vision, In Algorithms in Algebraic Geometry and Applications, Prog. in Math., 143:285-306, eds. L. González and T. Recio. Birkhäuser, Basel, (1996). 
6. Schönflies, A., Geometrie der Bewegung in synlhetischer Darstellung. B. G. Teubner, Leipzig, (1886). \{ see also the review of this book by F. Morley in Bull. Amer. Math. Soc. 5(10):476-480 (1899). \}

7. Selig, J.M., Geometric Fundamentals of Robotics, Springer Verlag, New York, (2005).

8. Selig, J.M., Quadratic Constraints on Rigid-Body Displacements, ASME Jounal of Mechanisms and Robotics, 2:041009 (7 pages), (2010).

9. Wampler, C.W., Forward displacement analysis of general six-in-parallel sps (Stewart) platform manipulators using soma coordinates. Mech. Mach. Theory 31(3): 331-337, (1996).

10. Wampler, C.W., On a rigid body subject to point-plane constraints, ASME J. of Mechanical Design, 128(1):151-158, (2006).

11. Zsombor-Murray, P.J. and Gfrerrer, A., A unified approach to direct kinematics of some reduced motion parallel manipulators. ASME Journal of Mechanisms and Robotics, 2(2):021006 (10 pages), (2010). 